\title{
Preface to the special volume on the third Sandia Fracture Challenge
}

\author{
Sharlotte L. B. Kramer
}

Published online: 25 June 2019

(C) This is a U.S. government work and not under copyright protection in the U.S.; foreign copyright protection may apply 2019

The ever-growing reliance on computational simulations to predict all aspects of the lifecycle of a mechanical system, from fabrication to failure, has prompted the mechanics community to self-assess its abilities to perform those predictions. Benchmark problems in mechanics that compare simulations that use different computational approaches with experiments have sprung up lately, including the NIST AM-Bench looking at additively manufactured (AM) materials (https://www.nist.gov/ambench), the Contact-Mechanics Challenge (Müser et al. 2017) considering adhesion between two nominally flat surfaces, Numisheet providing semiannual benchmarking activities in sheet metal forming (http://numisheet2018.org), and the Sandia Fracture Challenge (SFC) (Boyce et al. 2014, 2016) investigating ductile failure. The previous SFCs have demonstrated that progress has been made in computations of ductile failure but improvements still

\footnotetext{
Sandia National Laboratories is a multimission laboratory managed and operated by National Technology and Engineering Solutions of Sandia, LLC, a wholly owned subsidiary of Honeywell International Inc., for the U.S. Department of Energy's National Nuclear Security Administration under contract DE-NA0003525. Any subjective views or opinions that might be expressed in this paper do not necessarily represent the views of the U.S. Department of Energy or the United States Government.
}

S. L. B. Kramer $(\varangle)$

Sandia National Laboratories, Albuquerque, NM, USA

e-mail: slkrame@sandia.gov can be made, hence the third Sandia Fracture Challenge (SFC3), the subject of this Special Volume. The most recent installment of SFC builds on previous successes and tackles the difficult problem of fracture in an AM 316L stainless steel structure.

The SFC3 was preceded by internal Sandia National Laboratories' assessments [Report number SAND20116801]; the first Sandia Fracture Challenge (SFC1) issued in 2012, reported in a 2014 Special Volume of the International Journal of Fracture [Vol. 186, Nos. 1-2, March/April 2014]; and the second Sandia Fracture Challenge (SFC2) issued in 2014, reported in a 2016 Special Volume of the International Journal of Fracture [Vol. 198, Nos. 1-2, March/April 2016]. SFC1 asked for predictions of failure in a $15-5 \mathrm{PH}$ stainless steel plate with holes around a blunt notch, which experimentally resulted in two different crack paths due to issues with geometric tolerances in the features. The comparison of the blind predictions and the experiments revealed: (1) most teams could predict early elasto-plastic behavior; (2) no consensus on failure model or numerical implementation was found; (3) the provided standard calibration data of tensile and fracture toughness tests were deemed insufficient to predict crack initiation, particularly for shear-dominated failure; and, (4) no team accounted for geometric tolerance uncertainties that could have shown the bifurcation of failure modes that were experimentally observed. SFC2 considered fracture in a rate-dependent Ti-6Al$4 \mathrm{~V}$ structure with notches and holes tested at two different displacement rates. This challenge showed that 
in general the predictions were improved compared with SFC1 but revealed new sources of discrepancies: (1) boundary conditions, representing surface contact and friction; (2) plasticity model, accounting for sheet anisotropy and Lode angle dependence; (3) coupling, estimating thermal work coupling factor; (4) failure criteria, choosing a realistic failure parameter; and (5) damage progression and fracture morphology. The general features of predictions that did well in SFC2 included: (1) boundary conditions representing the clevis-pin connection, generally contiguous pin with translation/rotation or contact pin; (2) an anisotropic yield function; and (3) utilization of both tensile and shear data. There was no consensus approach amongst the more successful teams on thermal coupling, plastic hardening, failure criterion, or damage evolution, which was true in SFC1. Also shown in both SFC1 and SFC2 is that a prerequisite for a good prediction of ductile failure is a good calibration of plastic behavior. These two previous challenges demonstrated that ductile failure predictions can be good or bad for a multitude of interdependent reasons from computational choices to type of experimental calibration data, thus, the need for additional Challenge scenarios to identify areas of research and improvement.

The SFC3 was designed to push the envelope of the previous Challenges by comparing the computations and experiments using local measures, requesting measures of uncertainty in the predictions, utilizing AM metal that has large inherent variability compared to wrought metal, and providing micro-computed tomography (micro-CT) scans of the Challenge specimens to show the geometric variability and void content. Additionally, in SFC3 the Challenge geometry was not a set of extruded 2D features in a plate like the previous Challenges, but rather a 3D structure with internal features that could only be produced via AM processes. For the Challenge, the teams were provided with more information than ever before, including the micro-CT scans of the Challenge specimens and geometric measurements of the feature based on the scans, tension and notched tensions tests of 316L SS specimens built on the same build-plate as the Challenge geometry, electron backscatter diffraction (EBSD) information on grain texture, and post-test fractography of the calibration specimens. Also provided was information about the experimental parameters of the Digital Image Correlation (DIC) metrology used during the Challenge scenario testing against which the predictions would be compared. The quantities of interest ranged from the more standard global load versus displacement curves to strain contours across lines of interest on the Challenge specimens. The Challenge was issued to the international mechanics community through iMechanica.org and through direct e-mail to known groups with potential interest. The Challenge information is available via the Materials Data Facility (Kramer et al. 2018). The volunteer teams were given seven months to return their predictions, longer than prior Challenges, due to complex nature of the AM metal.

This Special Volume begins with a lead article that describes SFC3 and its outcome. The lead article details the Challenge, the experimental calibration data provided, the blind predictions including their methodologies, a comparison of the predictions to the experimental results, a discussion of sources of discrepancy and areas for improvement, and appendices that provide more detail about the predictions, additional plots for comparing the predictions and experiments, and additional post-blind experiments performed. This extensive article delves into the elements of relatively successful predictions and identified sources of discrepancies. This lead article was made possible by the contributions from its 52 co-authors from 16 institutions who were the experimental and computational mechanicians for SFC3. This article was facilitated by largegroup meetings via WebEx and six section leaders, Drs. Brad Boyce, Ashley Spear, Amanda Jones, Jakob Ostien, James Sobotka, and Sharlotte Kramer. Several months of email correspondence and dedication to the topic produced this lead article that represents all of the participants' assessment of SFC3. In addition to the lead article, this Special Volume includes ten individual articles from prediction teams; all teams were offered the opportunity to submit an article for this Special Volume. Another individual article is an experimental reinvestigation of the Challenge scenario to consider the evolution of damage in the Challenge geometry. All articles included in this Special Volume were subject to the customary peer review process of the Journal.

The SFC is only possible with the volunteer efforts of computational researchers around the world. More than 50 participants from 16 institutions contributed their time and resources to support this effort, testing their skills and approaches against the same problem. They were given limited time to predict a difficult problem and then spent countless hours to support the post-blind assessment of their predictions against the 
experiments in the name of learning and improvement not only for themselves, but for the greater mechanics community. These participants deserve our gratitude.

The SFC would also not be possible without a great team of experimentalists, dedicated to quality and detailed work. In Sandia's Structural Mechanics Lab, Dr. Amanda Jones, Jhana Gearhart, Darren Pendley, David Johnson, Colin McConnell, and Dr. Sharlotte Kramer performed Challenge geometry testing. In Sandia's Material Mechanics Lab, Bradley Salzbrenner and Dr. Brad Boyce performed all the base material testing and corroboration tests of the Challenge geometry. In Sandia's Radiography lab, Andrew Lentfer and Carl Jacques performed the micro-CT scans. Also at Sandia, Bonnie McKenzie and Dr. Joseph Michael performed fractography imaging and EBSD imaging, respectively. Prof. K. Ravi-Chandar at University of Texas at Austin and Dr. Thomas Tancogne-Dejean at Massachusetts Institute of Technology performed additional, post-blind calibration experiments on the $316 \mathrm{~L}$ SS material.

I would like to acknowledge the support at Sandia National Laboratories and beyond. Drs. James Redmond and Eliot Fang supported the SFC through funding from the DOE Advanced Scientific Computing (ASC) and through their encouragement to pursue the SFC. Dr. Dennis Croessmann, Darrick Jones, and Dr. Scott Peterson provided financial support for the experimental effort through the NNSA Weapon System Engineering and Assessment Technology (WSEAT) and Delivering Environments (DE) Engineering Campaigns. We received support from Dr. Benjamin Blaiszik at the Materials Data Facility with archiving the SFC3 data. Prof. K. Ravi-Chandar has played an instrumental role in the SFC as a computational participant in prior Challenges, post-blind experimentalist in SFC3, keen peer-reviewer of our SFC3 efforts during the post-blind assessment, and editor-inchief of the International Journal of Fracture. Without his support, this Special Volume would not be possible.

This third installment of SFC demonstrates an enduring effort by the mechanics community for selfassessment in the area of ductile failure. Together, we have shown some progress over the three Challenges and have identified many areas where research is required in ductile failure:
- Stochastic and local nature of ductile failure,

- Accepted shear-dominated experiments for model calibration,

- Theoretical and experimental work on thermomechanical coupling,

- Effect of material anisotropy on failure,

- Efficient constitutive and failure models for large structures,

- Quantitative comparison of full-field experimental and computational data, and

- Methods for uncertainty quantification on engineering time-scales.

The success of the SFC to identify areas for improvements in computational and experimental methods for ductile failure has spurred the formation of a new venture in Challenge-type research in structural mechanics: the Structural Reliability Partnership (SRP). The purpose of the SRP is to coordinate research, share best practices, and leverage investments from multiple institutions on areas of mutual interest in the domain of structural reliability. At present, there are 17 member institutions bringing $\$ 4.2 \mathrm{M}$ in leveraged funding to the Partnership. In addition to hosting annual workshops and coordinating material exchange, the SRP is planning to sponsor Challenge problems in different areas of structural reliability, broadening the scope of the original SFC. The first two SRP Challenges are being designed and will be released soon in the areas of reliability in additively manufactured structures and hydrogen-assisted fracture in welded pipelines. For more information about the SRP or to become a member, you can contact Brad Boyce (blboyce@sandia.gov) or visit www.sandia.gov/srp.

\section{References}

Boyce BL, Kramer SLB, Fang HE, Cordova TE, Neilsen MK, Dion K et al (2014) The Sandia Fracture Challenge: blind round robin predictions of ductile tearing. Int J Fract 186:568

Boyce BL, Kramer SLB, Bosiljevac T, Corona E, Moore J, Elkhodary K et al (2016) The second Sandia Fracture Challenge: predictions of ductile failure under quasi-static and moderate-rate dynamic loading. Int J Fract 198:5-100

Kramer S, Boyce B, Jones A, Gearhart J, Salzbrenner B (2018) The third Sandia Fracture Challenge. https://doi.org/10. 18126/M26D20 
Müser MH, Dapp WB, Bugnicourt R, Sainsot P, Lesaffre N, Lubrecht TA, Persson BNJ, Harris K, Bennett A, Schulze K, Rohde S, Ifju P, Sawyer WG, Angelini T, Esfahani HA, Kadkhodaei M, Akbarzadeh S, Wu J-J, Vorlaufer G, Vernes A, Solhjoo S, Vakis AI, Jackson RL, Xu Y, Streator J, Rostami A, Dini D, Medina S, Carbone G, Bottiglione F, Afferrante L, Monti J, Pastewka L, Robbins MO, Greenwood
JA (2017) Meeting the contact-mechanics challenge. Tribol Lett 65:118

Publisher's Note Springer Nature remains neutral with regard to jurisdictional claims in published maps and institutional affiliations. 